\title{
Intragroup and Intracluster Light
}

\section{J. Christopher Mihos}

\author{
Department of Astronomy, Case Western Reserve University
}

\begin{abstract}
The largest stellar halos in the universe are found in massive galaxy clusters, where interactions and mergers of galaxies, along with the cluster tidal field, all act to strip stars from their host galaxies and feed the diffuse intracluster light (ICL) and extended halos of brightest cluster galaxies (BCGs). Studies of the nearby Virgo Cluster reveal a variety of accretion signatures imprinted in the morphology and stellar populations of its ICL. While simulations suggest the ICL should grow with time, attempts to track this evolution across clusters spanning a range of mass and redshift have proved difficult due to a variety of observational and definitional issues. Meanwhile, studies of nearby galaxy groups reveal the earliest stages of ICL formation: the extremely diffuse tidal streams formed during interactions in the group environment.
\end{abstract}

Keywords. galaxies: clusters, galaxies: halos, galaxies: evolution

\section{Introduction}

In considering the connection between accretion and the formation of galaxy halos, perhaps nowhere is the process more dramatically illustrated than in the assembly of the most massive halos - the extended BCG envelopes and diffuse intracluster light (ICL) that is found in the centers of massive galaxy clusters. Unlike quiescent field galaxies whose major accretion era lies largely in the past, under hierarchical accretion scenarios, clusters of galaxies are the most recent objects to form (e.g. Fakhouri et al. 2010); their massive central galaxies continue to undergo active assembly and halo growth even at the current epoch, and may have accreted as much as half their mass since a redshift of $z=0.5$ (e.g. de Lucia \& Blaizot 2007). Thus the cluster environment presents an ideal locale for studying the accretion-driven growth of massive galaxy halos.

As galaxy clusters assemble, their constituent galaxies interact with one another, first within infalling groups, then inside the cluster environment itself. Over the course of time, a variety of dynamical processes liberate stars from their host galaxies, forming and feeding the growing population of intracluster stars. This complex accretion history is illustrated in Figure 1, using the collisionless simulations of Rudick et al. (2011). At early times, individual galaxies are strewn along a collapsing filament of the cosmic web. Gravity quickly draws these galaxies into small groups, which then fall together to form larger groups. In the group environment, slow interactions between galaxies lead to strong tidal stripping and the formation of discrete tidal tails and streams. As the groups fall into the cluster, this material is efficiently mixed into the cluster ICL (Rudick et al. 2006, 2009). Concurrently, mergers of galaxies in the cluster core expel more stars into intracluster space (Murante et al. 2007), as does ongoing stripping of infalling galaxies due to interactions both with other cluster galaxies and with the cluster potential itself (Conroy et al. 2007, Purcell et al. 2007, Contini et al. 2014). Additionally, even in-situ star formation in the intracluster medium, from gas stripped from infalling galaxies, may contribute some fraction of the ICL as well (Puchwein et al. 2010). All these processes lead to a continual growth of the intracluster light over time, as clusters continue to be fed by infalling groups and major cluster mergers. This evolution predicts that ICL properties should be linked to the dynamical state of the cluster - early in their formation 


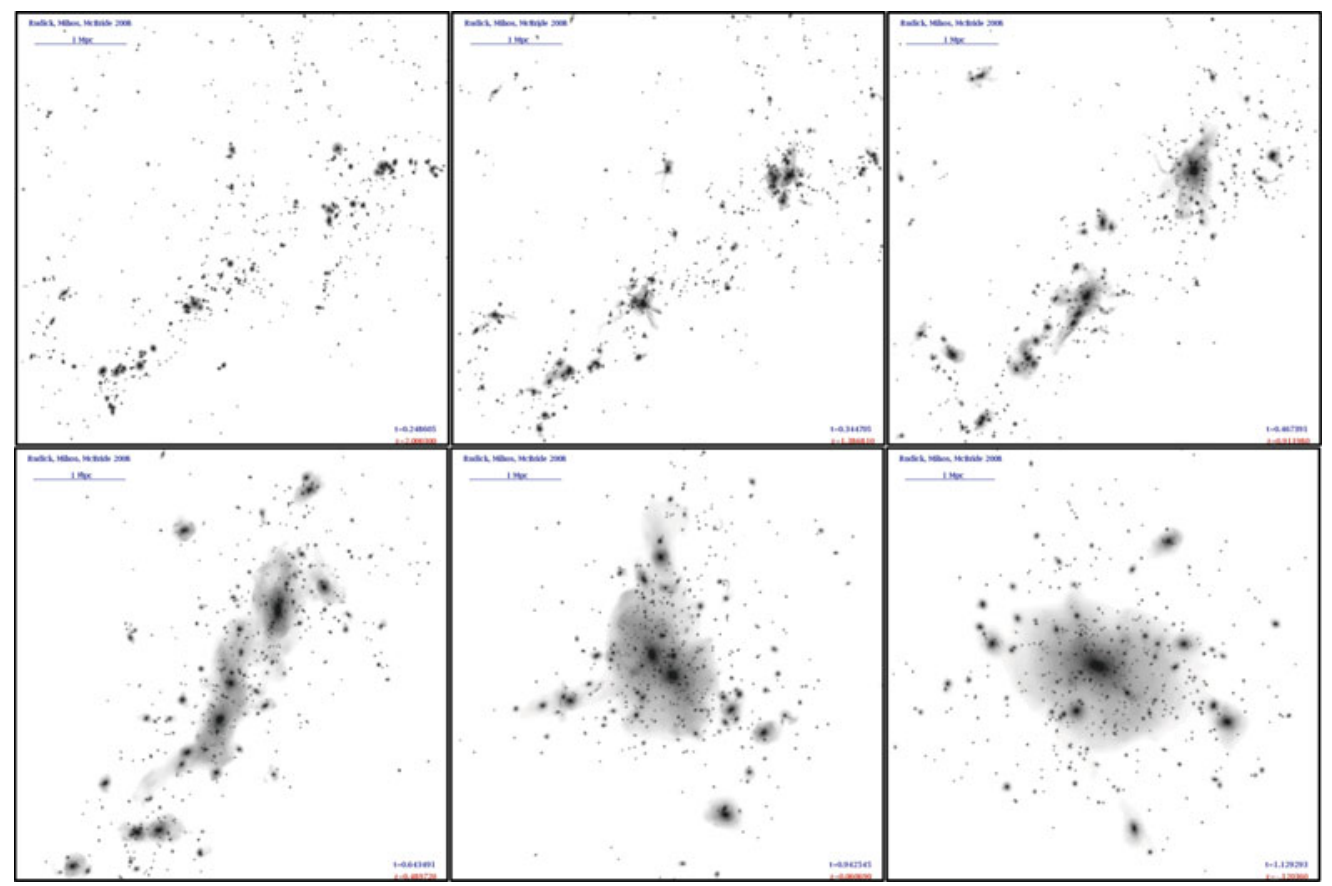

Figure 1. Formation of intracluster light during the assembly of a $10^{15} M_{\odot}$ galaxy cluster. The panels run from $z=2$ (upper left) to the present day (lower right). From Rudick et al. (2011).

history, clusters should be marked by a low total ICL fraction but with a high proportion of light in cold (and more easily visible tidal streams), while more evolved clusters would have higher ICL fractions found largely in a smooth, diffuse, and well-mixed state.

The fact that these various processes all operate concurrently makes it difficult to isolate their individual contributions to the ICL, and computational studies differ on whether group accretion, major mergers, or tidal stripping dominate the ICL. Fortunately, these processes imprint a variety of observable signatures in the ICL. The morphology and color of the diffuse light as well as the spatial distribution and kinematics of discrete ICL tracers (red giant branch (RGB) stars, planetary nebulae (PNe), and globular clusters (GCs)) all have potential to disentangle the ICL formation channels. For example, the galaxy mass-metallicity relationship predicts that stripping of low mass satellites would deposit preferentially metal-poor stars into the ICL, while mergers of massive galaxies would lead to more metal-rich ICL. Similarly the age distribution of ICL populations may differentiate between stripping of old stellar systems versus that from star-forming galaxies, or even contributions from in-situ ICL production. Thus, observational studies of the morphology, colors, kinematics, and stellar populations in the ICL are well-motivated to track the detailed accretion histories of massive clusters.

\section{ICL in the Virgo Cluster}

We can use the nearby Virgo Cluster to illustrate the wealth of information locked in the ICL. Figure 2 shows deep, wide-field imaging of the Virgo Cluster taken using CWRU Astronomy's Burrell Schmidt telescope (Mihos et al. in prep). Covering 16 square degrees down to a surface brightness of $\mu_{V} \sim 28.5$, the imaging reveals the complex web of diffuse light spread throughout the core of Virgo. A number of tidal streams are visible, most 


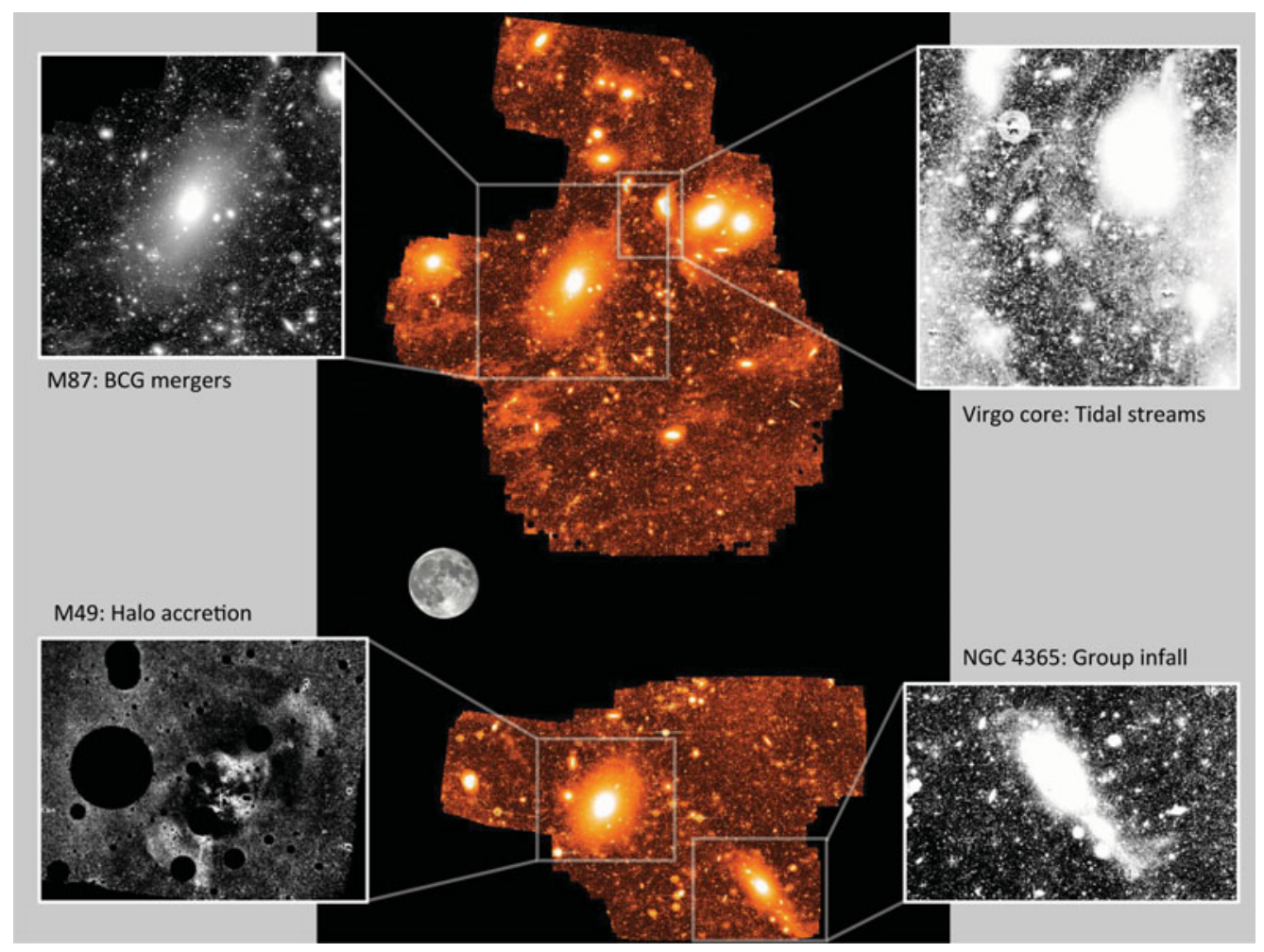

Figure 2. Diffuse light in the Virgo Cluster. The center panel shows deep $\left(\mu_{V, \lim } \approx 28.5\right)$ wide-field imaging of Virgo taken using CWRU's Burrell Schmidt telescope (Mihos et al. in prep); the inset moon shows a $30^{\prime}$ scale. Panels show M87's extended halo (upper left; Mihos et al. 2005), tidal streams in the Virgo core (upper right; Mihos et al. 2005, 2015), M49's system of accretion shells (lower left; Janowiecki et al. 2010, Mihos et al. 2013) and the diffuse intragroup light surrounding NGC 4365 (lower right; Bogdán et al. 2012, Mihos et al. in prep).

notably two long (> $100 \mathrm{kpc}$ ) thin streams NW of M87 (Mihos et al. 2005, Rudick et al. 2010). Smaller streams are also found around the Virgo ellipticals M86 and M84, likely due to stripping of low mass satellite galaxies, as well as a system of shells and plumes around M89 suggestive of one or more major mergers (Malin 1979, Janowiecki et al. 2010). However, the total luminosity contained in these discrete streams is only $\sim 1-2 \times 10^{9} \mathrm{~L}_{\odot}$; the bulk of the ICL is likely found in more diffuse form, locked in the extended halo of M87 or strewn throughout the cluster at lower surface brightness.

Indeed the deep imaging reveals not only the thin ICL streams but also the large radial extent of the halos of Virgo ellipticals. In particular, M87's halo is traced beyond 150 $\mathrm{kpc}$, where a variety of signatures indicative of past accretion events can be seen. The outermost regions of M87's halo are extremely boxy (Mihos et al. in prep), a behavior reflected in the spatial distribution of its GC system as well (Durrell et al. 2014). This combination of boxy isophotes and low halo rotation (Romanowsky et al. 2012) hints at a major merger event in M87's past, and indeed, both the GC and PNe systems around M87 show kinematic substructure (Romanowsky et al. 2012 and Longobardi et al. 2015a respectively), suggesting the recent accretion of one or more $\sim 10^{10} \mathrm{~L}_{\odot}$ systems.

Signatures of past accretion are also found in other Virgo ellipticals as well. Located south of the Virgo core, M49 has long been known to have a dynamically complex halo, as traced by kinematic substructure in its GC system (Côté et al. 2003). The deep imaging 
in Figure 2 reveals the cause: after subtraction of a smooth isophotal model for M49, an extensive set of accretion shells (Janowiecki et al. 2010, Arrigoni Battaia et al. 2011, Capaccioli et al. 2015) can be seen, spanning $\sim 150 \mathrm{kpc}$ in extent and containing close to $10^{9} \mathrm{~L}_{\odot}$ of light (Janowiecki et al. 2010). The shells are morphologically similar to those formed during the radial accretion of a low mass satellite, and may be linked to the tidally disturbed dwarf companion VCC 1249 (Arrigoni Battaia et al. 2011). The shells are also distinctly redder than M49's surrounding halo (Mihos et al. 2013), suggesting that the accretion event is building up both the mass and metallicity of M49's outer halo.

Figure 2 also illustrates the efficacy of the group environment in driving ICL formation. Lying $5.3^{\circ}$ to the SW of the Virgo core (and $\sim 7 \mathrm{Mpc}$ behind; Mei et al. 2007) is the infalling Virgo $\mathrm{W}^{\prime}$ group, with the massive elliptical NGC 4365 at its core. Our deep imaging shows an extended, diffuse tidal tail emanating SW from the galaxy (Bogdán et al. 2012; Mihos et al. in prep), and GC kinematics clearly link the tail to an interaction with its companion NGC 4342 (Blom et al. 2014). The tail contains $\sim 1.5 \times 10^{9} \mathrm{~L}_{\odot}$, and a number of other streams are visible in NGC 4365's halo as well (including the loop visible to the NE of the galaxy), all indicative of cold tidal stripping in the group environment. Once the $\mathrm{W}^{\prime}$ group eventually falls into the main body of Virgo, this diffuse and extended intragroup light will be easily mixed into Virgo's diffuse ICL.

Finally, the imaging contains a dramatic example of the complex dynamical interplay between tidal stripping, ICL formation, and the destruction and formation of cluster galaxy populations. Lying at the center of the "Tidal streams" panel of Figure 2 is a large and extremely dim ultra-diffuse galaxy; with a half light radius of $9.7 \mathrm{kpc}$ and central surface brightness $\mu_{V}=27.0$ it is the most extreme ultradiffuse cluster galaxy yet discovered (Mihos et al. 2015). The galaxy also sports a long tidal tail arcing $\sim 100 \mathrm{kpc}$ to the north, as well as a compact nucleus whose photometric properties are well-matched to those of ultracompact dwarf galaxies (UCDs) found in Virgo (e.g. Zhang et al. 2015, Liu et al. 2015). In this object, we are clearly seeing the tidal destruction of a low mass, nucleated galaxy which is both feeding Virgo's ICL population and giving rise to a new Virgo UCD.

To go beyond morphology and study the stellar populations in Virgo's ICL in detail, a variety of tools are available. The colors of the streams around M87 ( $B-V=0.7-1.0$; Rudick et al. 2010) are well-matched to those of the Virgo dE population and of M87's halo itself, suggesting M87's halo may be built at least in part from low mass satellite accretion. HST imaging of discrete RGB populations in Virgo intracluster fields shows the ICL to be predominantly old and metal-poor $(t>10 \mathrm{Gyr},[\mathrm{Fe} / \mathrm{H}] \approx-1$; Williams et al. $2007)$, but with an additional population of stars with intermediate ages and higher metallicities $(t \approx 4-8 \mathrm{Gyr},[\mathrm{Fe} / \mathrm{H}] \gtrsim-0.5)$. These younger populations may arise either from stripped star forming galaxies or from ICL formed in-situ. The inference that stripping of late-type galaxies has contributed to the Virgo ICL is also supported by the luminosity function of PNe in M87's outer halo, which shows a "dip" characteristic of lower mass galaxies with extended star formation histories (Longobardi et al. 2015b). The diversity of stellar populations seen in Virgo's ICL almost certainly reflects the diversity of processes that create diffuse light in clusters.

\section{ICL Systematics: Challenges and Metrics}

While Virgo's proximity gives us a detailed view of intracluster stellar populations, to gain a wider census of ICL in galaxy clusters we must move beyond Virgo. Going to greater distances opens up the ability to study ICL in a wider sample of clusters which span a range of mass, dynamical state, and redshift, allowing us to connect ICL 


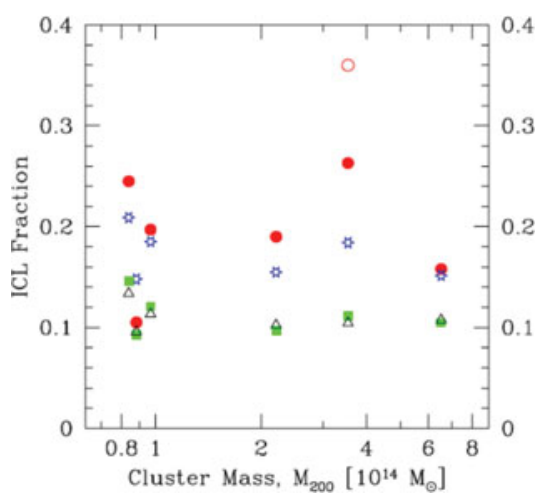

Figure 3. ICL fractions in simulated galaxy clusters, taken from Rudick et al. (2011). Five simulated clusters of varying mass are shown; at a given mass, the symbols show ICL fractions calculate for a single cluster using different ICL definitions: stars at low surface brightness ( $\mu_{V}>26.5$, green squares), stars in low density intracluster space now (open triangles) or ever (blue stars), stars unbound from galaxies (filled red circles), including stars kinematically separated from the central cD galaxy (open red circle). See Rudick et al. (2011) for details.

properties with cluster evolution. This comes at a cost, however; beyond Virgo, current generation telescopes cannot directly image intracluster stars, and even studies of more luminous tracers such as PNe and GC become more difficult. At higher redshifts, one becomes limited to broadband imaging, where the strong cosmological $(1+z)^{4}$ surface brightness dimming makes the already diffuse ICL even more difficult to observe.

Aside from these observational difficulties, a second major problem is the ambiguous definition of intracluster light itself. Since much of the ICL is formed via the mergers that build up the central BCG, there is often no clear differentiation between the BCG halo and the extended ICL - the two components blend smoothly together (and indeed may not be conceptually distinct components at all). In attempts to separate BCG halos from extended ICL, a variety of photometric definitions have been proposed, which typically adopt different functional forms (such as multiple $r^{1 / 4}$ or Sersic profiles) for each component when fitting the total profile (e.g. Gonzalez et al. 2005, Krick \& Bernstein 2007, Seigar et al. 2007). However, such definitions are very sensitive to the functional forms adopted for the profiles. For example, M87's profile is reasonably well-fit by either a single Sersic or a double $r^{1 / 4}$ model (Janowiecki et al. 2010); the former fit would imply little additional ICL, while the latter fit puts equal light into the inner and outer profiles. To avoid this ambiguity, alternate non-parametric measures have also been employed to characterize the ICL luminosity, defining the ICL as diffuse light fainter than some characteristic surface brightness (e.g. Feldmeier et al. 2004, Burke et al. 2015). While these present systematic uncertainties of their own, simulations suggest that thresholds of $\mu_{V} \gtrsim 26.5$ do a reasonable job of separating out an extended and perhaps unrelaxed ICL component from the central BCG light (Rudick et al. 2011, Cooper et al. 2015).

Still other methods propose kinematic separation of the ICL from the central galaxy light. Dolag et al. (2010) used simulated clusters to show that separate kinematic populations exist in cluster cores, well-characterized by distinct Maxwellian distributions. These kinematic populations then separate out spatially into two Sersic-like profiles plausibly identified as the BCG galaxy and the cluster ICL (perhaps reflecting different accretion events as well; Cooper et al. 2015). And indeed these definitions have some observational support. Longslit spectroscopy of the BCG galaxy in Abell 2199 shows a velocity dispersion profile that first falls with radius, then increases in the outer halo to join smoothly 
onto the cluster velocity dispersion (Kelson et al. 2002). Meanwhile in Virgo the velocities of the PNe around M87 show a double Gaussian distribution (Longobardi et al. 2015b), suggesting distinct BCG and ICL components. However, observational constraints make accessing kinematic information for the ICL in distant clusters a daunting task.

A comparison of these different metrics is shown in Figure 3 (from Rudick et al. 2011), which shows that the inferred ICL fraction in simulated clusters can vary by factors of 2-3 depending on the adopted metric (see also Puchwein et al. 2010). Kinematic separation leads to higher ICL fractions, as a significant amount of starlight found within the BCG galaxy belongs to the high-velocity ICL component. In contrast, density-based estimates yield systematically lower ICL fractions, as material at high surface brightness is typically assigned to the cluster galaxies independent of its kinematic properties.

Given both the ambiguity in defining the ICL and the observational difficulties in studying it, attempts to characterize ICL in samples of clusters spanning a range of mass and redshift have led to varying results. An early compilation of results for local clusters by Ciardullo et al. (2004) showed ICL fractions ranging from $\sim 15-40 \%$, with no clear dependence on cluster velocity dispersion or Bautz-Morgan type. Recent imaging of more distant clusters probes the connection between cluster evolution and ICL more directly, but again yields mixed results. While Guennou et al. (2012) find no strong difference between the ICL content of clusters between at $z \sim 0.5$ and today, Burke et al. (2015) find rapid evolution in the ICL fraction of massive clusters over a similar redshift range. Other studies of clusters at $z \sim 0.3-0.5$ yield ICL fractions of 10-25\% (Presotto et al. 2014, Montes \& Trujillo 2014, Giallongo et al. 2014), similar to $z=0$ results. However, these studies use different ICL metrics and are limited to only a handful of clusters; clearly a large sample of clusters with ICL fractions measured in a consistent manner is needed to tackle the complex question of ICL evolution.

A similar story holds for recent attempts to constrain ICL stellar populations as well. Using HST imaging of distant CLASH clusters, DeMaio et al. (2015) infer moderately low metallicities $([\mathrm{Fe} / \mathrm{H}] \sim-0.5)$ from the ICL colors, in contrast to the case of Abell 2744, where Montes \& Trujillo (2014) use colors to argue for a dominant population of intermediate age stars with solar metallicity. Meanwhile, spectroscopic population synthesis studies show similarly diverse results. For example, in the Hydra I cluster, Coccato et al. (2011) find old ICL populations with sub-solar metallicities, while in the massive cluster RX J0054.0-2823, Melnick et al. (2012) find similarly old but metal-rich ICL stars $([\mathrm{Fe} / \mathrm{H}] \gtrsim 0)$. However, while intriguing, all these studies are subject to strong photometric biases, limited largely to the brightest portions of the ICL which may not be representative of the ICL as a whole and may also include substantial fraction of what would normally be considered BCG light as well.

\section{Diffuse Light in Nearby Galaxy Groups}

The evolution shown in Figure 1 argues that interactions in the group environment should be particularly effective at stripping stars from galaxies and redistributing them into the diffuse intragroup light, an important precursor to the ICL in massive clusters. Curiously, though, these arguments have not always been borne out observationally. In the nearby Leo I group, searches for intragroup light using both PNe (Castro-Rodriguez et al. 2003) and broadband imaging (Watkins et al. 2014) have come up empty, particularly notable given that the system is contains a large $(\sim 200 \mathrm{kpc}) \mathrm{HI}$ ring thought to be collisional in origin (Michel-Dansac et al. 2010). Similarly, the M101 group also show little sign of extended diffuse light (Mihos et al. 2013), despite the tidal disturbances evident in M101 and its nearby companions. Even in the clearly interacting M81/M82 


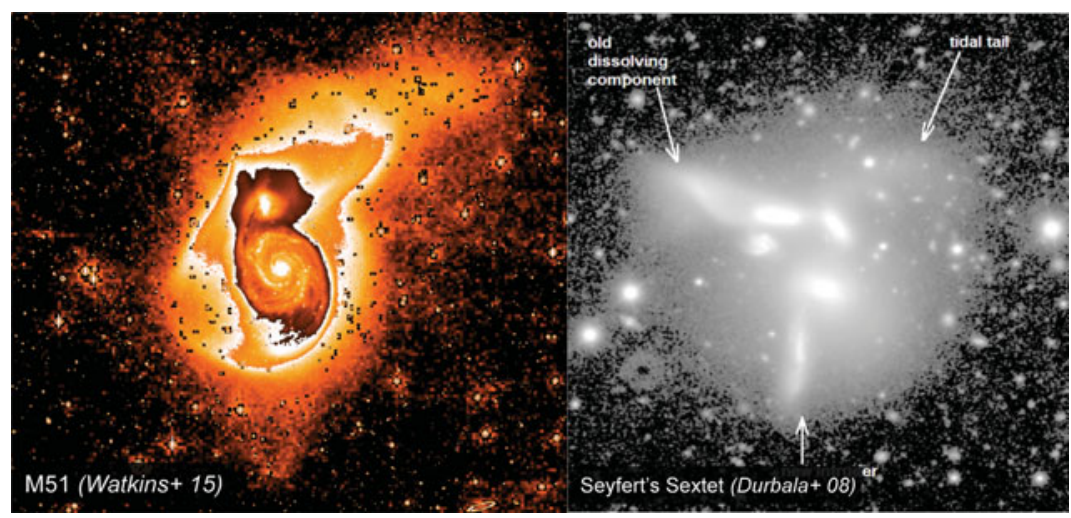

Figure 4. Left: Deep imaging of M51 showing diffuse tidal debris extending to nearly $50 \mathrm{kpc}$ (Watkins et al. 2015). Right: Diffuse light in Seyfert's Sextet (Durbala et al. 2008).

group, early searches for orphaned RGB stars (Durrell et al. 2004) and PNe (Feldmeier et al. 2003) could only place upper limits on the intragroup light fraction $(\lesssim 2 \%)$.

In contrast, intragroup light is quite evident in dense, strongly interacting groups. The ability for these strong interactions to expel diffuse material to large distances is shown in recent deep imaging of the M51 system by Watkins et al. (2015; Fig 4a), where several extremely low surface brightness plumes extend nearly $50 \mathrm{kpc}$ from the center. Similarly, many compact groups are awash in diffuse light (e.g. Da Rocha et al. 2005, 2008), including the archetypal groups Seyfert's Quintet (Mendes de Oliveira et al. 2001) and Seyfert's Sextet (Durbala et al. 2008, Figure 4b). The contrast between the copious diffuse light seen in these dense systems and the dearth of light in loose groups is striking, arguing either that the tidal debris is rapidly dispersed to even lower (undetectable) surface brightnesses, or that close interactions in loose groups are relatively uncommon.

More recently, the ability to probe discrete stellar populations in external galaxies provides a powerful new tool for studying intragroup light. Probing stellar densities far below the capabilities of wide-area surface photometry, these techniques are now revealing the diffuse light contained even in loose groups. Deep imaging by Okamoto et al. (2015) has uncovered the previously undetected and very extended stellar tidal debris field in the M81 group, while imaging of M31 and M33 by the PAndAS team (e.g. Ibata et al. 2014) has mapped the myriad of tidal streams that characterize Andromeda's extended stellar halo and trace its past interaction with M33. While at low surface brightness and containing only a small amount of the total light of their parent groups, the diffuse starlight found in these studies of nearby loose groups represent the important first step in building the intragroup and intracluster light in dense galaxy environments.

\section{References}

Arrigoni Battaia, F., Gavazzi, G., Fumagalli, M., et al. 2012, A\&A, 543, A112

Blom, C., Forbes, D. A., Foster, C., et al. 2014, MNRAS, 439, 2420

Bogdán, Á., Forman, W. R., Zhuravleva, I., et al. 2012, ApJ, 753, 140

Burke, C., Collins, C. A., Stott, J. P., \& Hilton, M. 2012, MNRAS, 425, 2058

Burke, C., Hilton, M., \& Collins, C. 2015, MNRAS, 449, 2353

Côté, P., McLaughlin, D. E., Cohen, J. G., \& Blakeslee, J. P. 2003, ApJ, 591, 850

Capaccioli, M., Spavone, M., Grado, A., et al. 2015, A\&A, 581, A10

Castro-Rodríguez, N., Aguerri, J. A. L., Arnaboldi, M., et al. 2003, A\&SA, 405, 803 
Ciardullo, R., Mihos, J. C., Feldmeier, J. J., Durrell, P. R., \& Sigurdsson, S. 2004, Recycling Intergalactic and Interstellar Matter, 217, 88

Coccato, L., Gerhard, O., Arnaboldi, M., \& Ventimiglia, G. 2011, A\&3A, 533, A138

Conroy, C., Wechsler, R. H., \& Kravtsov, A. V. 2007, ApJ, 668, 826

Contini, E., De Lucia, G., Villalobos, Á., \& Borgani, S. 2014, MNRAS, 437, 3787

Cooper, A. P., Gao, L., Guo, Q., et al. 2015, MNRAS, 451, 2703

Da Rocha, C. \& Mendes de Oliveira, C. 2005, MNRAS, 364, 1069

De Lucia, G. \& Blaizot, J. 2007, MNRAS, 375, 2

DeMaio, T., Gonzalez, A. H., Zabludoff, et al. 2015, MNRAS, 448, 1162

Dolag, K., Murante, G., \& Borgani, S. 2010, MNRAS, 405, 1544

Durbala, A., del Olmo, A., Yun, M. S., et al. 2008, AJ, 135, 130

Durrell, P. R., Decesar, M. E., Ciardullo, R., Hurley-Keller, D., \& Feldmeier, J. J. 2004, Recycling Intergalactic and Interstellar Matter, 217, 90

Durrell, P. R., Côté, P., Peng, E. W., et al. 2014, ApJ, 794, 103

Fakhouri, O., Ma, C.-P., \& Boylan-Kolchin, M. 2010, MNRAS, 406, 2267

Feldmeier, J. J., Durrell, P. R., Ciardullo, R., \& Jacoby, G. H. 2003, Planetary Nebulae: Their Evolution and Role in the Universe, 209, 605

Feldmeier, J. J., Mihos, J. C., Morrison, H. L., et al. 2004, ApJ, 609, 617

Ferrarese, L., Côté, P., Cuillandre, J.-C., et al. 2012, ApJS, 200, 4

Giallongo, E., Menci, N., Grazian, A., et al. 2014, ApJ, 781, 24

Gonzalez, A. H., Zabludoff, A. I., \& Zaritsky, D. 2005, ApJ, 618, 195

Guennou, L., Adami, C., Da Rocha, C., et al. 2012, A\&AA, 537, A64

Ibata, R. A., Lewis, G. F., McConnachie, A. W., et al. 2014, ApJ, 780, 128

Janowiecki, S., Mihos, J. C., Harding, P., et al. 2010, ApJ, 715, 972

Kelson, D. D., Zabludoff, A. I., Williams, K. A., et al. 2002, ApJ, 576, 720

Krick, J. E. \& Bernstein, R. A. 2007, AJ, 134, 466

Liu, C., Peng, E. W., Cote, P., et al. 2015, arXiv:1508.07334

Longobardi, A., Arnaboldi, M., Gerhard, O., \& Mihos, J. C. 2015, A\&3A, 579, L3

Longobardi, A., Arnaboldi, M., Gerhard, O., \& Hanuschik, R. 2015, A\& A, 579, A135

Malin, D. F. 1979, Nature, 277, 279

Mei, S., Blakeslee, J. P., Côté, P., et al. 2007, ApJ, 655, 144

Melnick, J., Giraud, E., Toledo, I., Selman, F., \& Quintana, H. 2012, MNRAS, 427, 850

Mendes de Oliveira, C., Plana, H., Amram, P., Balkowski, C., \& Bolte, M. 2001, AJ, 121, 2524

Michel-Dansac, L., Duc, P.-A., Bournaud, F., et al. 2010, ApJ Letters, 717, L143

Mihos, J. C., Durrell, P. R., Ferrarese, L., et al. 2015, ApJ Letters, 809, L21

Mihos, J. C., Harding, P., Feldmeier, J., \& Morrison, H. 2005, ApJ Letters, 631, L41

Mihos, J. C., Harding, P., Rudick, C. S., \& Feldmeier, J. J. 2013, ApJ Letters, 764, L20

Mihos, J. C., Harding, P., Spengler, C. E., Rudick, C. S., \& Feldmeier, J. J. 2013, ApJ, 762, 82

Montes, M. \& Trujillo, I. 2014, ApJ, 794, 137

Murante, G., Giovalli, M., Gerhard, O., et al. 2007, MNRAS, 377, 2

Okamoto, S., Arimoto, N., Ferguson, A. M. N., et al. 2015, ApJ Letters, 809, L1

Presotto, V., Girardi, M., Nonino, M., et al. 2014, A\&A, 565, A126

Puchwein, E., Springel, V., Sijacki, D., \& Dolag, K. 2010, MNRAS, 406, 936

Purcell, C. W., Bullock, J. S., \& Zentner, A. R. 2007, ApJ, 666, 20

Romanowsky, A. J., Strader, J., Brodie, J. P., et al. 2012, ApJ, 748, 29

Rudick, C. S., Mihos, J. C., Frey, L. H., \& McBride, C. K. 2009, ApJ, 699, 1518

Rudick, C. S., Mihos, J. C., Harding, P., et al. 2010, ApJ, 720, 569

Rudick, C. S., Mihos, J. C., \& McBride, C. 2006, ApJ, 648, 936

Rudick, C. S., Mihos, J. C., \& McBride, C. K. 2011, ApJ, 732, 48

Seigar, M. S., Graham, A. W., \& Jerjen, H. 2007, MNRAS, 378, 1575

Watkins, A. E., Mihos, J. C., \& Harding, P. 2015, ApJ Letters, 800, L3

Watkins, A. E., Mihos, J. C., Harding, P., \& Feldmeier, J. J. 2014, ApJ, 791, 38

Williams, B. F., Ciardullo, R., Durrell, P. R., et al. 2007, ApJ, 656, 756

Zhang, H.-X., Peng, E. W., Côté, P., et al. 2015, ApJ, 802, 30 